\title{
Oral Hygiene Practices and their Socio-demographic Correlates among Adults in Burkina Faso: Results from the First National Survey
}

Jeoffray Diendéré ( $\nabla$ jeoffray.diendere@gmail.com )

Research Institute for Health Sciences (IRSS)

\section{Seydou Ouattara}

Université Joseph Ki-Zerbo

Jean Kaboré

Research Institute for Health Sciences (IRSS)

Ibrahim Traoré

Université Joseph Ki-Zerbo

Augustin Nawidimbasba Zeba

Research Institute for Health Sciences (IRSS)

Séni Kouanda

Research Institute for Health Sciences (IRSS)

\section{Research Article}

Keywords: Oral health, Oral hygiene practices, Sociodemographic factors, Education, WHO-STEPS, Burkina Faso.

Posted Date: October 14th, 2021

DOI: https://doi.org/10.21203/rs.3.rs-952612/v1

License: (1) This work is licensed under a Creative Commons Attribution 4.0 International License. Read Full License 


\section{Abstract}

Background. Socio-demographic parameters are the driving determinants for oral hygiene practices. This purpose aims to describe oral hygiene practices and associated socio-demographic factors in Burkinabè population, using the first nationally-representative data.

Methods. This descriptive, cross-sectional study included 4677 adults through multistage cluster sampling performed during the first WHO STEPS survey conducted in 2013 in Burkina Faso. The practices we considered were the frequencies of tooth cleaning, the fluoridated toothpaste use and a dentist visit within the last six months. Sociodemographic variables and oral hygiene practices were described and the first ones were used as the explanatory variables for the seconds in the multivariable analyses.

Results. Individuals who cleaned teeth at least once a day represented $82.8 \%$ and at least twice a day $31.4 \%$; those who used fluoridated toothpaste were $25.6 \%$ and $2.1 \%$ have visited a dentist. With the highest oddsratio, only being educated was a favorable factor for each of oral hygiene practices. Living in an urban area or being younger adults were favorable factors to the daily repetitive tooth cleaning or the use of a fluoridated paste. Female gender applied more to a daily repetitive cleaning, as well as to the dentist visit.

Conclusion. Cleaning teeth at least once a day was common among Burkinabè while cleaning at least twice a day, the use of fluoridated paste or dentist visiting were infrequent. Education was the key favorable determinant for healthy oral hygiene practices and improving oral health literacy interventions through basic health education should be promoted.

\section{Background}

Chronic diseases are a growing burden to people, to health-care systems and to societies, and the its rapid increase (in burden) is particularly prevalent in the low and middle-income countries (LMICs) [1]. Oral disorders have been ranked among the three leading level 3 causes in terms of incident cases were since 1990 [2]. The good oral hygiene practices are efficient to prevent oral diseases [3] including oral and digestive cancers [4,5] or cardiovascular risks [3]. Both oral health outcomes and access to oral health services are positively correlated with the number of oral health professionals as dental hygienists [6]. However, SubSaharan African (SSA) LMICs are a short supply of skilled human resources for oral health [7] with the wide socioeconomic inequalities in this sector [8]; and sociodemographic factors are the crucial determinants for oral hygiene practices or health $[9,10]$.

The World Health Organization (WHO) mentioned the need for the vast majority of countries to establish a surveillance system for measuring progress in the control of oral disease and promotion of oral health [11]. The standardized tool designed by this health organization for the surveillance of the non-communicable diseases in LMICs, the Stepwise approach to surveillance (WHO STEPS), includes a specific section on the oral health status and related behaviors [12]. This section specifically explores oral hygiene practices such as the frequencies of tooth cleaning, the use of fluoridated toothpaste and the visit to a dentist. The first survey using this tool was performed in 2013 in Burkina Faso. In this low-income country, the population basedstudies on oral health or hygiene are scare. Nevertheless, it has been reported dental plaque and calculus in respectively in $53 \%$ and $80 \%$ of adults of $25-54$ y, living in the East of the country [13] since 1989. In 2018, 
there were only 28 dental surgeons in the country [14]. The objective of our purpose was to describe oral hygiene practices and associated socio-demographic factors among Burkinabè adults, using nationally and representative data provided by the first WHO STEPS survey.

\section{Methods}

\section{Study design}

A secondary cross-sectional analysis was performed using data from the first WHO STEPS [12] survey conducted in 2013 in Burkina Faso. This study is a recommended tool for surveillance of chronic diseases and their risk factors. The survey is a standardized method to collect, analyze and disseminate data. It includes a representative sample of the study population, which allows the results to be generalizable to the entire population.

Ethics considerations: The protocol of the STEPS survey was approved by the Ethics Committee for Health Research of the Ministry of Health of Burkina Faso (deliberation No: 2012-12092; December 05, 2012). Written informed consent was systematically obtained from each participant in the STEPS survey.

\section{Sampling}

The nationally representative sample size was calculated to be 4800 adults aged $25-64$ years, according to the WHO STEPS methodology [12]. This sample size (4800) considered the estimates by age group and sex and achieved sufficient accuracy by weighting the numbers of age groups for each gender. It was also weighted to ensure representativeness with regard to the living environment.

Data collection and variables of interest: Data were collected from a questionnaire and direct measurement. Data collection was conducted from 3 September to 24 October 2013. The data were collected using standardized WHO STEPS questionnaires input into laptop computers. Sociodemographic information was recorded via face-to-face interviews in the language spoken by the participant. Participants' demographic variables included living environment, gender, age, marital status, education level, and occupation. The oral hygiene practices were: the frequencies of tooth cleaning, the use of fluoridated toothpaste and the visit to the dentist.

\section{Individuals included for our secondary analyses}

We studied variables of individuals with complete data with regard to sociodemographic parameters and hygiene practices specified above. Of the sample of 4800 individuals surveyed, 105 were not eligible; 10 and eight had missing data concerning sociodemographic variables and dentist visit respectively and thus, we included 4677 participants for our secondary analyses.

\section{Statistical analyses}

We used StataCorp Stata Statistical Software for Windows (Version 12.0, College Station, Texas, US) to analyse the data. The quantitative variables were expressed as the means \pm standard deviations, and the categorical variables were expressed as percentages (\%). The chi-squared test was used to compare 
categorical variables. Logistic regression analysis was performed to identify the sociodemographic factors with each the four practices. The regression model was determined by backward elimination (i.e., the progressive elimination of nonsignificant factors by decreasing order of significance). For all analyses, a pvalue below 0.05 was considered significant.

\section{Results}

Of the sample studied, nine (0.2\%) individuals had no teeth and amongst the rest, 82.8\% (81.6-83.8) cleaned teeth at least once a day and 31.4\% (30.1-32.7) at least twice a day. Two hundred forty-seven (5.3\%) ignored if they used a fluoridated toothpaste while $24.2 \%$ (23.0-254) declared to use a fluoridated toothpaste. At overall, only $2.1 \%$ (1.7-2.6) have visited a dentist the last six months. Table 1 reports the distribution of oral hygiene practices by socio-demographic characteristics and Table 2 summarizes results of stepwise logistic regressions. The associated factors with cleaning teeth at least twice a day were high education level $(\mathrm{aOR}=4.0, \mathrm{p}<0.001)$, living in an urban area $(\mathrm{aOR}=1.6, \mathrm{p}<0.001)$, professions with regular income $(\mathrm{aOR}=1.6$, $p<0.001)$, being woman $(a O R=1.4, p<001)$ or married/cohabiting $(a O R=1.4, p<0.01)$. Associated factors with the use of a fluoridated paste were high level of education ( $a O R=6.7, p<0.001)$, living in an urban area $(a O R=2.4, p<0.001)$ and being young adults $(a O R=1.4, p<0.001)$. The favorable factors for visiting dentists were high education level $(a O R=5.7, p<0.001)$ and being woman $(a O R=1.6, p<0.05)$, while being young was a negative factor $(\mathrm{aOR}=0.5, \mathrm{p}<0.05)$. 
Table 1

Distribution of oral hygiene practices by socio-demographic characteristics

\section{Socio- demographic Oral hygiene practices characteristics}

$\begin{array}{lllll}\begin{array}{l}\mathrm{N} \text { (un- } \\ \text { weighted } \\ \%)\end{array} & \begin{array}{l}\text { Visiting dentist } \\ \text { within the last } 6 \\ \text { months }\end{array} & \begin{array}{l}\text { Cleaning teeth at } \\ \text { least once a day \% } \\ (95 \% \mathrm{Cl})\end{array} & \begin{array}{l}\text { Cleaning } \\ \text { teeth at least } \\ \text { twice a day }\end{array} & \begin{array}{l}\text { Use of } \\ \text { fluoridated } \\ \text { toothpaste }\end{array} \\ \mathbf{N = \mathbf { 4 6 7 7 }} & \mathbf{N}=\mathbf{4 6 7 7} & \mathbf{N}=\mathbf{4 6 6 8 +} & \mathbf{N}=\mathbf{4 6 6 8 +} & \mathbf{N}=\mathbf{4 4 2 1 \ddagger}\end{array}$

Age ranges (in

years, y)

\begin{tabular}{|c|c|c|c|c|c|}
\hline$-25-30 y$ & $\begin{array}{l}1306 \\
(27.9)\end{array}$ & $1.7(1.1-2.5)$ & 85.8 (83.8-87.7) & $\begin{array}{l}36.4(33.8- \\
39.0)\end{array}$ & $\begin{array}{l}28.3(25.8- \\
30.9)\end{array}$ \\
\hline$-30-44 y$ & $\begin{array}{l}1988 \\
(42.5)\end{array}$ & $2.2(1.6-2.9)$ & $85.1(83.4-86.6)$ & $\begin{array}{l}31.7(29.6- \\
33.8)\end{array}$ & $\begin{array}{l}27.4(25.4- \\
29.5)\end{array}$ \\
\hline$-45-64 y$ & $\begin{array}{l}1383 \\
(29.6)\end{array}$ & $2.5(1.8-3.5)$ & 76.4 (74.1-78.6) & $\begin{array}{l}26.2(23.9- \\
28.6)\end{array}$ & $\begin{array}{l}20.1(17.9- \\
22.5)\end{array}$ \\
\hline$p$-value & & NS & $\star \star \star$ & $\star \star \star$ & $\star \star \star$ \\
\hline \multicolumn{6}{|l|}{ Gender } \\
\hline - Men & $\begin{array}{l}2245 \\
(48.0)\end{array}$ & $1.8(1.3-2.4)$ & $83.1(81.5-84.6)$ & $\begin{array}{l}29.9(28.0- \\
31.8)\end{array}$ & $\begin{array}{l}29.0(27.1- \\
31.0)\end{array}$ \\
\hline - Women & $\begin{array}{l}2432 \\
(52.0)\end{array}$ & $2.5(1.9-3.2)$ & $82.4(80.8-83.9)$ & $\begin{array}{l}32.8(30.9- \\
34.7)\end{array}$ & $\begin{array}{l}22.4(20.7- \\
24.2)\end{array}$ \\
\hline$p$-value & & NS & NS & * & $\star \star \star$ \\
\hline \multicolumn{6}{|l|}{ Residence area } \\
\hline - Rural & $\begin{array}{l}3734 \\
(79.8)\end{array}$ & $1.6(1.3-2.1)$ & $80.3(79.0-81.6)$ & $\begin{array}{l}28.3(26.8- \\
29.7)\end{array}$ & $\begin{array}{l}19.6 \text { (18.3- } \\
21.0)\end{array}$ \\
\hline - Urban & $\begin{array}{l}943 \\
(20.2)\end{array}$ & $4.1(3.0-5.6)$ & $92.3(90.4-93.9)$ & $\begin{array}{l}43.7(40.5- \\
46.9)\end{array}$ & $\begin{array}{l}48.5 \text { (45.2- } \\
51.8)\end{array}$ \\
\hline$p$-value & & $\star \star \star$ & $\star \star \star$ & $\star \star \star$ & $\star \star \star$ \\
\hline \multicolumn{6}{|l|}{ Education level } \\
\hline $\begin{array}{l}\text { - No formal/no } \\
\text { education }\end{array}$ & $\begin{array}{l}3618 \\
(77.4)\end{array}$ & $1.5(1.1-1.9)$ & $80.2(78.8-81.5)$ & $\begin{array}{l}28.3(26.9- \\
29.8)\end{array}$ & $\begin{array}{l}18.6(17.3- \\
19.9)\end{array}$ \\
\hline - Primary achieved & $\begin{array}{l}727 \\
(15.5)\end{array}$ & $3.2(2.0-4.7)$ & 88.4 (85.9-90.7) & $\begin{array}{l}31.1(27.7- \\
34.6)\end{array}$ & $\begin{array}{l}37.2(33.7- \\
40.9)\end{array}$ \\
\hline $\begin{array}{l}\text { - Secondary or } \\
\text { more }\end{array}$ & $332(7.1)$ & $6.9(4.4-10.2)$ & 98.2 (96.1-99.3) & $\begin{array}{l}65.4(60.0- \\
70.5)\end{array}$ & $\begin{array}{l}72.5(67.4- \\
77.2)\end{array}$ \\
\hline
\end{tabular}

†The nine individuals without tooth were not included in the analyzes; $\ddagger$ Individuals without tooth (nine) or those who did not know if their toothpaste was or not fluoridated (247), were not included in this analyze; NS: Non-significant p-value; *p-value <0.05; **: p-value < 0.01; ***: p-value $<0.001$. 


\begin{tabular}{|c|c|c|c|c|c|}
\hline \multirow{2}{*}{$\begin{array}{l}\begin{array}{l}\text { Socio- } \\
\text { demographic } \\
\text { characteristics }\end{array} \\
\text { p-value }\end{array}$} & \multicolumn{5}{|c|}{ Oral hygiene practices } \\
\hline & & 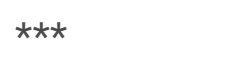 & $\star \star \star ~$ & $\star \star \star ~$ & $\star \star \star ~$ \\
\hline \multicolumn{6}{|l|}{ Occupation } \\
\hline $\begin{array}{l}\text { - Occupation with } \\
\text { regular income }\end{array}$ & $\begin{array}{l}3497 \\
(74.8)\end{array}$ & $1.9(1.5-2.4)$ & 83.1 (81.8-84.3) & $\begin{array}{l}32.5(31.0- \\
34.1)\end{array}$ & $\begin{array}{l}25.3\left(23.8^{-}\right. \\
26.8)\end{array}$ \\
\hline $\begin{array}{l}\text { - Job with not } \\
\text { regular income }\end{array}$ & $\begin{array}{l}1180 \\
(25.2)\end{array}$ & $2.8(1.9-3.9)$ & $81.7(79.4-83.9)$ & $\begin{array}{l}28.0(25.5- \\
30.7)\end{array}$ & $\begin{array}{l}26.5(24.0- \\
29.2)\end{array}$ \\
\hline$p$-value & & $\star \star \star ~$ & $\star \star \star \star ~$ & $\star \star \star \star ~$ & $\star \star \star ~$ \\
\hline \multicolumn{6}{|l|}{ Marital status } \\
\hline - Singles & $\begin{array}{l}642 \\
(13.7)\end{array}$ & $2.5(1.4-4.0)$ & $82.6(81.4-83.7)$ & $\begin{array}{l}30.5(26.9- \\
34.2)\end{array}$ & $\begin{array}{l}33.9(30.2- \\
37.9)\end{array}$ \\
\hline Married/cohabiting & $\begin{array}{l}4035 \\
(86.3)\end{array}$ & $2.1(1.7-2.6)$ & $83.8(80.7-86.5)$ & $\begin{array}{l}31.5(30.1- \\
33.0)\end{array}$ & $\begin{array}{l}24.3(23.0- \\
25.7)\end{array}$ \\
\hline$p$-value & & NS & NS & NS & 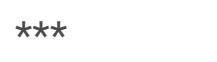 \\
\hline \multicolumn{6}{|c|}{$\begin{array}{l}\text { TThe nine individuals without tooth were not included in the analyzes; } \neq \text { Individuals without tooth (nine) or or } \\
\text { those who did not know if their toothpaste was or not fluoridated ( } 247) \text {, were not included in this analyze; } \\
\text { NS: Non-significant p-value; *p-value }<0.05 ; * \star \text {; } p \text {-value }<0.01 ; * \star * \text {-value }<0.001 \text {. }\end{array}$} \\
\hline
\end{tabular}


Table 2

Socio-demographic correlates of oral hygiene practices, in logistic regressions

\begin{tabular}{|c|c|c|c|c|c|c|c|c|}
\hline & \multicolumn{2}{|c|}{$\begin{array}{l}\text { Visited a dentist } \\
\text { within the last } 6 \\
\text { months ( } \mathrm{N}= \\
4677)\end{array}$} & \multicolumn{2}{|c|}{$\begin{array}{l}\text { Cleaning teeth at } \\
\text { least once a day }(\mathrm{N} \\
=4668 \mathrm{t})\end{array}$} & \multicolumn{2}{|c|}{$\begin{array}{l}\text { Cleaning teeth at } \\
\text { least twice a day } \\
(\mathrm{N}=4668 \mathrm{t})\end{array}$} & \multicolumn{2}{|c|}{$\begin{array}{l}\text { Use of fluoridated } \\
\text { toothpaste }(\mathrm{N}= \\
4421 \neq)\end{array}$} \\
\hline & $\begin{array}{l}\mathrm{COR} \\
(95 \% \\
\mathrm{Cl})\end{array}$ & $\begin{array}{l}\text { aOR } \\
(95 \% \\
\mathrm{Cl})\end{array}$ & $\begin{array}{l}\mathrm{COR} \\
(95 \% \\
\mathrm{Cl})\end{array}$ & $\begin{array}{l}\text { aOR } \\
(95 \% \\
\mathrm{Cl})\end{array}$ & $\begin{array}{l}\mathrm{cOR} \\
(95 \% \\
\mathrm{Cl})\end{array}$ & $\begin{array}{l}\text { aOR } \\
(95 \% \\
\mathrm{Cl})\end{array}$ & $\begin{array}{l}\text { COR } \\
(95 \% \\
\mathrm{Cl})\end{array}$ & $\begin{array}{l}\text { aOR } \\
(95 \% \\
\text { Cl) }\end{array}$ \\
\hline \multicolumn{9}{|l|}{ Gender } \\
\hline - Men & 1 & 1 & 1 & 1 & 1 & 1 & 1 & 1 \\
\hline - Women & $\begin{array}{l}1.4 \\
(0.9- \\
2.1)\end{array}$ & $\begin{array}{l}1.6 \\
(1.1- \\
2.5)^{\star}\end{array}$ & $\begin{array}{l}0.9(0.8- \\
1.1)\end{array}$ & $\begin{array}{l}>1.0 \\
(0.9- \\
1.2)\end{array}$ & $\begin{array}{l}>1.0 \\
(1.1- \\
1.3)^{\star}\end{array}$ & $\begin{array}{l}1.4 \\
(1.2- \\
1.6)^{\star \star \star}\end{array}$ & $\begin{array}{l}0.7(0.6- \\
0.8)^{\star \star \star}\end{array}$ & $\begin{array}{l}0.7 \\
(0.6- \\
0.8)^{\star \star \star}\end{array}$ \\
\hline \multicolumn{9}{|l|}{ Place of Residence } \\
\hline - Rural & 1 & 1 & 1 & 1 & 1 & 1 & 1 & 1 \\
\hline - Urban & $\begin{array}{l}2.6 \\
(1.7- \\
3.9)^{\star \star \star}\end{array}$ & $\begin{array}{l}1.4 \\
(0.9- \\
2.3)\end{array}$ & $\begin{array}{l}2.9(2.3- \\
3.8)^{\star \star \star}\end{array}$ & $\begin{array}{l}2.2(1.6- \\
2.8)^{\star \star \star}\end{array}$ & $\begin{array}{l}2.0 \\
(1.7- \\
2.3)^{\star \star \star}\end{array}$ & $\begin{array}{l}1.6 \\
(1.3- \\
1.9)^{\star \star \star}\end{array}$ & $\begin{array}{l}3.9(3.3- \\
4.5)^{\star \star \star}\end{array}$ & $\begin{array}{l}2.4 \\
(2.0- \\
2.8)^{\star \star \star}\end{array}$ \\
\hline \multicolumn{9}{|l|}{ Age (in years, y) } \\
\hline$-45-64 y$ & 1 & 1 & 1 & 1 & 1 & 1 & 1 & 1 \\
\hline$-30-44 y$ & $\begin{array}{l}0.9 \\
(0.5- \\
1.3)\end{array}$ & $\begin{array}{l}0.7 \\
(0.5- \\
1.1)\end{array}$ & $\begin{array}{l}1.8(1.5- \\
2.1)^{\star \star \star}\end{array}$ & $\begin{array}{l}1.7(1.4- \\
2.0)^{\star \star \star}\end{array}$ & $\begin{array}{l}1.3 \\
(1.1- \\
1.5)^{\star \star}\end{array}$ & $\begin{array}{l}1.2 \\
(1.1- \\
1.4)^{\star}\end{array}$ & $\begin{array}{l}1.5(1.3- \\
1.8)^{\star \star \star}\end{array}$ & $\begin{array}{l}1.4 \\
(1.2- \\
1.7)^{\star \star \star}\end{array}$ \\
\hline$-25-30 y$ & $\begin{array}{l}0.7 \\
(0.4- \\
1.1)\end{array}$ & $\begin{array}{l}0.5 \\
(0.3- \\
0.9)^{\star}\end{array}$ & $\begin{array}{l}1.9(1.5- \\
2.3)^{\star \star \star}\end{array}$ & $\begin{array}{l}1.8(1.4- \\
2.2)^{\star \star \star}\end{array}$ & $\begin{array}{l}1.6 \\
(1.4- \\
1.9)^{\star \star \star}\end{array}$ & $\begin{array}{l}1.5 \\
(1.3- \\
1.8)^{\star \star \star}\end{array}$ & $\begin{array}{l}1.6(1.3- \\
1.9)^{\star \star \star}\end{array}$ & $\begin{array}{l}1.4 \\
(1.2- \\
1.8)^{\star \star \star}\end{array}$ \\
\hline \multicolumn{9}{|l|}{ Education level } \\
\hline $\begin{array}{l}\text { - Not formal / not } \\
\text { education }\end{array}$ & 1 & 1 & 1 & 1 & 1 & 1 & 1 & 1 \\
\hline - Primary & $\begin{array}{l}2.2 \\
(1.3- \\
3.5)^{\star \star}\end{array}$ & $\begin{array}{l}2.5 \\
(1.5- \\
4.1)^{\star \star \star}\end{array}$ & $\begin{array}{l}1.9(1.5- \\
2.4)^{\star \star \star}\end{array}$ & $\begin{array}{l}1.5(1.1- \\
1.9)^{\star \star}\end{array}$ & $\begin{array}{l}1.1 \\
(0.9- \\
1.4)\end{array}$ & $\begin{array}{l}1.1 \\
(0.9- \\
1.2)\end{array}$ & $\begin{array}{l}2.6(2.2- \\
3.1)^{\star \star \star}\end{array}$ & $\begin{array}{l}1.9 \\
(1.6- \\
2.3)^{\star \star \star}\end{array}$ \\
\hline $\begin{array}{l}\text { - Secondary or } \\
\text { more }\end{array}$ & $\begin{array}{l}4.9 \\
(3.0- \\
8.1)^{\star \star \star}\end{array}$ & $\begin{array}{l}5.7 \\
(3.4- \\
9.4)^{\star \star \star}\end{array}$ & $\begin{array}{l}13.4 \\
(6.0- \\
30.3)^{\star \star \star}\end{array}$ & $\begin{array}{l}8.1(3.5- \\
18.4)^{\star \star \star *}\end{array}$ & $\begin{array}{l}4.8 \\
(3.8- \\
6.1)^{\star \star \star}\end{array}$ & $\begin{array}{l}4.0 \\
(3.1- \\
5.2)^{\star \star \star}\end{array}$ & $\begin{array}{l}11.6 \\
(8.9- \\
15.0)^{\star \star \star}\end{array}$ & $\begin{array}{l}6.7 \\
(5.1- \\
8.9)^{\star \star \star}\end{array}$ \\
\hline
\end{tabular}

†The nine individuals without tooth were not included in the analyzes; $\ddagger$ Individuals without tooth (nine) or those who did not know if their toothpaste was or not fluoridated (247), were not included in this analyze. Stars indicate the significant p-values only and *: P-value $<0.05$, $* *$ : P-value $<0.01, * \star *$ : P-value $<0.001$; aOR: adjusted odds-ratio; cOR: crude Odds-ratio. 


\begin{tabular}{|c|c|c|c|c|c|c|c|c|}
\hline \multirow[b]{2}{*}{$\begin{array}{l}\text { - Job with } \\
\text { inconstant } \\
\text { income/jobess }\end{array}$} & \multicolumn{2}{|c|}{$\begin{array}{l}\text { Visited a dentist } \\
\text { within the last } 6 \\
\text { months }(\mathrm{N}= \\
\text { 4677) }\end{array}$} & \multicolumn{2}{|c|}{$\begin{array}{l}\text { Cleaning teeth at } \\
\text { least once a day }(\mathrm{N} \\
=4668 \mathrm{t})\end{array}$} & \multicolumn{2}{|c|}{$\begin{array}{l}\text { Cleaning teeth at } \\
\text { least twice a day } \\
(\mathrm{N}=4668+)\end{array}$} & \multicolumn{2}{|c|}{$\begin{array}{l}\text { Use of fluoridated } \\
\text { toothpaste }(\mathrm{N}= \\
4421 \neq)\end{array}$} \\
\hline & 1 & 1 & 1 & 1 & 1 & 1 & 1 & 1 \\
\hline $\begin{array}{l}\text { - Occupation with } \\
\text { regular income }\end{array}$ & $\begin{array}{l}0.7 \\
(0.4- \\
1.0)\end{array}$ & $\begin{array}{l}0.8 \\
(0.5- \\
1.3)\end{array}$ & $\begin{array}{l}1.1(0.9- \\
1.3)^{(0-}\end{array}$ & $\begin{array}{l}1.2(1.1- \\
1.4)^{\star}\end{array}$ & $\begin{array}{l}1.2 \\
(1.1- \\
1.4)^{\star \star}\end{array}$ & $\begin{array}{l}1.6 \\
(1.4- \\
1.9)^{\star \star \star}\end{array}$ & $\begin{array}{l}0.9(0.8- \\
1.1)\end{array}$ & $\begin{array}{l}0.9 \\
(0.7- \\
1.1)\end{array}$ \\
\hline \multicolumn{9}{|l|}{ Marital status } \\
\hline - Singles & 1 & 1 & 1 & 1 & 1 & 1 & 1 & 1 \\
\hline - Married/cohabiting & $\begin{array}{l}0.8 \\
(0.5- \\
1.4)\end{array}$ & $\begin{array}{l}1.2 \\
(0.7- \\
2.1)\end{array}$ & $\begin{array}{l}0.9(0.7- \\
1.2)\end{array}$ & $\begin{array}{l}1.2(0.9- \\
1.5)\end{array}$ & $\begin{array}{l}1.1 \\
(0.9- \\
1.3)\end{array}$ & $\begin{array}{l}1.4 \\
(1.2- \\
1.7)^{\star \star}\end{array}$ & $\begin{array}{l}0.6(0.5- \\
0.8)^{\star \star \star}\end{array}$ & $\begin{array}{l}1.1 \\
(0.9- \\
1.3)\end{array}$ \\
\hline \multicolumn{9}{|c|}{$\begin{array}{l}\text { The nine individuals without tooth were not included in the analyzes; } ¥ \text { Individuals without tooth (nine) or or } \\
\text { those who did not know if their toothpaste was or not fluoridated }(247) \text {, were not included in this analyze. } \\
\text { Stars indicate the significant p-values only and *: P-value }<0.05 \text {, } \star * \text { : P-value }<0.01 \text {, } \star \star \star * \text { : P-value }<0.001 ; \\
\text { aOR: adjusted odds-ratio; cOR: crude Odds-ratio. }\end{array}$} \\
\hline
\end{tabular}

\section{Discussion}

The healthy oral hygiene practices were infrequently applied by Burkinabè adults and education was the key determinant for the good oral hygiene practices.

\section{Oral hygiene practices}

Majority of Burkinabè cleaned the teeth at least once a day (82.8\%) while the recommended number was at least twice a day [15]. Knowledge about oral hygiene was insufficient among Burkinabè [16] and can affect the level of consistent practices. Thus, only less than one-third cleaned the teeth at least twice a day as found in Indian adults (29\%) [17]. The use of fluoridated paste was reported by $25.6 \%$ whereas $18 \%$ in $35-44$ years old of Burkinabè a decade earlier [16]. Even if it appears to be an improvement, the personal oral hygiene in the absence of fluorides has not a benefit in terms of reducing of oral diseases as dental caries [18]. The fewer prevalence of those who visited a dentist $(2.1 \%)$ reflects the difficult access to oral care in low purchasing power area, and the insufficient human resource to face the need in terms of oral health $[7,19,16]$.

\section{Sociodemographic factor correlates with oral hygiene practices}

High education level: the correlates of high education level with each hygiene practice that we found were close to results reported among Nepalese adults [10], and among students, higher oral health literacy was associated with better oral health practices [20]. That may support why lower education was considered as risk factor for dental plaque or gingivitis [17]. Those with higher education levels usually become more concerned about their own physical or body appearance, including the tooth whiteness and then assume behaviors to this goal. It was found the positive effects of exposure to the oral health education program 
during primary school years on the oral health knowledge and practice [21] and we reported that just attending primary school was associated to the favorable oral hygiene practices (Table 2). The country would benefit from increasing primary school enrolment and completion rates (respectively at about $89 \%$ and $62 \%$ in 2019) [22], while integrating simple oral hygiene education modules into the national curriculum as it has been experienced in Bangladesh, Indonesia, Nepal and Tanzania [23]. Since the health literacy is a strong predictor of an individuals' health, health behavior and health outcomes [24], integrating oral and general health through health literacy practices which would be adapted for the general population, should be implemented [25].

Urban residency and young adults: Except for the practice of the dentist visit, other kinds of oral hygiene practices had an urban residency or being younger adults (25-30y or $30-44 y)$ as favorable factors, in line with the results of the Nepalese study [10]. In contrast to our study, it also reported the urban residency as the favorable factor to the dentist visit $(\mathrm{aOR}=1.9, \mathrm{p}<0.05)$, and suggest higher number of oral health professional in this country (1400 dentists, one per 20000 inhabitants), with a highest density of workers in an urban area [26]. Moreover, the specific source of motivations for cleaning teeth or toothpaste selection among young adults were the fear of losing of teeth and the whitening feature of teeth [27].

Female gender. concerning associations of the female gender with the good practices of cleaning teeth twice a day [aOR=1.4 (Cl:1.2-1.6)], as with visiting dentist [aOR=1.6 (1.1-2.5)], our report was similar to that among Nepalese people with the respective aOR of 1.7 (1.1-2.4) and 2.2 (1.2-3.8) [10]. In Burkina Faso, women represent at about $52.0 \%$ (as in our representative sample), and married/cohabiting Burkinabè $86.3 \%$ (Table 1). A simple educational intervention has a positive impact on oral health behaviors in groups [28] and in the framework of the family oral hygiene education for Burkinabè societies, women should be placed in key role. This is quite fitting, especially since being in group e.g., married/cohabiting was also associated with cleaning teeth at least twice a day [aOR=1.4 (1.2-1.7), Table 2].

Occupation with regular income: Professions with regular income was a favorable factor for cleaning teeth at least twice a day [aOR=1.6 (1.4-1.9), Table 2]. Professions may determine the income level, and high income among Korean adults was favorable for a daily repetitive tooth brushing [29]. High number of tooth cleaning may imply more financial investments to provide toothpaste while the share of Burkinabè people living on less than $\$ 1.90$ a day was $43.7 \%$ in 2014 [30]. In contrast to the Australian study reporting that an increased household income improved dental visiting [31], we noticed that even Burkinabè with regular income had not a favorable habit of dentist visit [(aOR=0.8 (0.5-1.3) Table 2]. It suggests a generalized low purchasing power to face dental care costs in Burkina Faso.

Limitations: Income variables were not collected and geographic data were not included in the analyzes and thus, we missed specific information on their impact in our multivariable models. While these first nationallyrepresentative data from 2013 may no longer reflect the current situation, they provide a relevant baseline that can be compared with future WHO STEPS survey data.

\section{Conclusion}


Cleaning teeth at least once a day was common among Burkinabè but not its daily repetition, or the use of the fluoridated paste or the habit of the dentist visit. Education was the key favorable determinant for a good oral hygiene practice. The country should increase primary school enrolment and completion rates, while integrating simple oral hygiene education modules into the national curriculum. Family oral hygiene education should be initiated, with the women in the key role. Meanwhile, improving access to fluoridated products and the training of dental human resources with their efficient density at the different stages of the national health care system should be undertaken.

\section{Abbreviations}

$\mathrm{aOR}$

Adjusted odds ratios

$\mathrm{Cl}$

Confidence interval

COR

Crude odds ratio

LMICs

Low and middle-income countries

NCDs

Non-communicable diseases

$\mathrm{OR}$

Odds ratio

SSA

Sub-Saharan-African

STEPS

STEPwise approach to Surveillance

WHO

World Health Organization.

\section{Declarations}

Ethics approval and consent to participate: The protocol of the STEPS survey was approved by the Ethics Committee for Health Research of the Ministry of Health (deliberation No: 2012-12092; December 05, 2012). Written informed consent was systematically obtained from each participant in the STEPS survey.

Consent for publication: Non applicable.

Availability of data: The database of the STEPS survey used for this secondary analysis is available at the Ministry of Health of Burkina Faso. It can be requested directed to Dr Brice Bicaba bicababrico78@gmail.com.

Competing interests: The authors declare that they have no competing interests.

Funding: None. 
Authors' contributions: J.D., S.O., J.K. and K.S. contributed to drafting the manuscript; J.D. and S.O. performed the statistical analysis; I.T. and AN.Z. initiated the first interpretation of the results; J.D., J.K. and K.S. reviewed the last version. All authors reviewed and approved the final manuscript.

Acknowledgments: The authors thank the Ministry of Health for providing them with the STEP survey database.

\section{References}

1. Petersen PE, Baehni PC. Periodontal health and global public health. Periodontol 2000. 2012;60:7-14.

2. GBD 2017 Disease and Injury Incidence and Prevalence Collaborators. Global, regional, and national incidence, prevalence, and years lived with disability for 354 diseases and injuries for 195 countries and territories, 1990-2017: a systematic analysis for the Global Burden of Disease Study 2017. Lancet. 2018;392:1789-858.

3. Park S-Y, Kim S-H, Kang S-H, Yoon C-H, Lee H-J, Yun P-Y, et al. Improved oral hygiene care attenuates the cardiovascular risk of oral health disease: a population-based study from Korea. Eur Heart J. 2019;40:113845 .

4. Gupta B, Bray F, Kumar N, Johnson NW. Associations between oral hygiene habits, diet, tobacco and alcohol and risk of oral cancer: A case-control study from India. Cancer Epidemiol. 2017;51:7-14.

5. Cordero OJ, Varela-Calviño R. Oral hygiene might prevent cancer. Heliyon. 2018;4:e00879.

6. Wing P, Langelier MH, Continelli TA, Battrell A. A Dental Hygiene Professional Practice Index (DHPPI) and access to oral health status and service use in the United States. J Dent Hyg. 2005;79:10.

7. Bhayat A, Chikte U. Human Resources for Oral Health Care in South Africa: A 2018 Update. Int J Environ Res Public Health. 2019;16.

8. Varenne B, Fournet F, Cadot E, Msellati P, Ouedraogo HZ, Meyer PE, et al. Environnement familial et disparités de santé dentaire des enfants en milieu urbain au Burkina Faso. Rev Epidemiol Sante Publique. 2011;59:385-92.

9. Jiménez R, Tapias-Ledesma MA, Gallardo-Pino C, Carrasco P, de Miguel AG. Influence of sociodemographic variables on use of dental services, oral health and oral hygiene among Spanish children. Int Dent J. 2004;54:187-92.

10. Thapa P, Aryal KK, Mehata S, Vaidya A, Jha BK, Dhimal M, et al. Oral hygiene practices and their sociodemographic correlates among Nepalese adult: evidence from non communicable diseases risk factors STEPS survey Nepal 2013. BMC Oral Health. 2016;16:105.

11. Petersen PE, Ogawa H. Strengthening the prevention of periodontal disease: the WHO approach. $J$ Periodontol. 2005;76:2187-93. 
12. World Health Organization. WHO steps surveillance manual: the WHO stepwise approach to chronic disease risk factor surveillance. Geneva: World Health Organization; 2005.

13. Abellard J, Decroix B, Kerebel LM. Enquête épidémiologique sur la santé bucco-dentaire à Fada N'Gourma (Burkina Faso). Bull Group Int Rech Sci Stomatol et Odontol. 1989;32:31-8.

14. Ministère de la Santé du Burkina Faso. Annuaire Statistique 2018 du Ministère de la Santé du Burkina Faso. 2019. http://cns.bf/IMG/pdf/annuaire_ms_2018.pdf. Accessed 20 Jan 2021.

15. Claydon NC. Current concepts in toothbrushing and interdental cleaning. Periodontol 2000. 2008;48:1022.

16. Varenne B, Petersen PE, Ouattara S. Oral health behaviour of children and adults in urban and rural areas of Burkina Faso, Africa. Int Dent J. 2006;56:61-70.

17. Sreenivasan PK, Prasad KVV, Javali SB. Oral health practices and prevalence of dental plaque and gingivitis among Indian adults. Clin Exp Dent Res. 2016;2:6-17.

18. Hujoel PP, Hujoel MLA, Kotsakis GA. Personal oral hygiene and dental caries: A systematic review of randomised controlled trials. Gerodontology. 2018;35:282-9.

19. Hau KP-H, Currie BL, Ng SP-Y, Le N, Poh CF-Y. Oral health status and possible explanatory factors of an inner-city low-income community. J Dent Sci. 2017;12:49-55.

20. Kanupuru KK, Fareed N, Sudhir KM. Relationship Between Oral Health Literacy and Oral Health Status Among College Students. Oral Health Prev Dent. 2015;13:323-30.

21. Alsumait A, ElSalhy M, Amin M. Long-Term Effects of School-Based Oral Health Program on Oral Health Knowledge and Practices and Oral Health-Related Quality of Life. Med Princ Pract. 2015;24:362-8.

22. Institut National de la Statistique et de la Démographie. Tableau de bord de la gouvernance édition 2019. 2019. http://www.insd.bf/n/contenu/pub_periodiques/tableaux_de_bord/TBG/TBG_2019.pdf. Accessed 18 Jan 2021.

23. Helderman W van P, Mikx F, Begum A, Adyatmaka A, Bajracharya M, Kikwilu E, et al. Integrating oral health into primary health care - experiences in Bangladesh, Indonesia, Nepal and Tanzania. IDJ. 2011;49:240-8.

24. Baskaradoss JK. Relationship between oral health literacy and oral health status. BMC Oral Health. 2018;18:172.

25. National Academies of Sciences, Engineering, and Medicine; Health and Medicine Division; Board on Population Health and Public Health Practice; Roundtable on Health Literacy. Integrating Oral and General Health Through Health Literacy Practices: Proceedings of a Workshop. Washington (DC): National Academies Press (US); 2019. http://www.ncbi.nlm.nih.gov/books/NBK544961/. Accessed 2 Feb 2021. 
26. Ministry of Health \& Population. Human Resource for Health: Nepal country profile, August 2013. 2013. http://www.nhssp.org.np/NHSSP_Archives/human_resources/HRH_Nepal_profile_august2013.pdf. Accessed 18 Jan 2021.

27. Özdoğan MS, Gümüşok M, Sariçam E. Attitudes and behavior regarding oral hygiene in a group university students: A self-reported. Selcuk Dent J. 2020. doi:10.15311/selcukdentj.545065.

28. Mattheus D, Shannon M, Lim E. Benefits of Oral Health Education at Women, Infant, and Children (WIC) Clinic Visits: Assessments of Parent's Oral Health Beliefs, Behaviors and Dental Access in O'ahu, Hawai'i. Hawaii J Health Soc Welf. 2020;79 5 Suppl 1:32-9.

29. Park J-B, Han K, Park Y-G, Ko Y. Association between socioeconomic status and oral health behaviors: The 2008-2010 Korea national health and nutrition examination survey. Exp Ther Med. 2016;12:2657-64.

30. World Bank Group. Poverty \& Equity Brief Burkina Faso Sub-Saharan Africa, April 2020. 2020. https://databank.worldbank.org/data/download/poverty/33EF03BB-9722-4AE2-ABC7AA2972D68AFE/Global_POVEQ_BFA.pdf. Accessed 17 Jan 2021.

31. Anikeeva O, Brennan DS, Teusner DN. Household income modifies the association of insurance and dental visiting. BMC Health Serv Res. 2013;13:432. 